\title{
Neurophysiological Reflex Mechanisms' Lack of Contribution to the Success of PNF Stretches
}

\author{
J. Brent Feland \\ brent_feland@byu.edu \\ Sterling C. Hilton \\ J. Ty Hopkins \\ tyhopkins@byu.edu \\ lain Hunter \\ iain_hunter@byu.edu \\ Ulrike H. Mitchell \\ Follow this and additional works at: https://scholarsarchive.byu.edu/facpub \\ dinextparthe for additional authors
}

\section{Original Publication Citation}

Mitchell UH, Myrer JW, Hopkins JT, Hunter I, Feland JB. (29). Neurophysiological reflex mechanisms lack of contribution to the success of PNF stretches, Journal of Sport

Rehabilitation, 18(3), 343-357.

\section{BYU ScholarsArchive Citation}

Feland, J. Brent; Hilton, Sterling C.; Hopkins, J. Ty; Hunter, lain; Mitchell, Ulrike H.; and Myrer, J. William, "Neurophysiological Reflex Mechanisms' Lack of Contribution to the Success of PNF Stretches" (2009). Faculty Publications. 871.

https://scholarsarchive.byu.edu/facpub/871

This Peer-Reviewed Article is brought to you for free and open access by BYU ScholarsArchive. It has been accepted for inclusion in Faculty Publications by an authorized administrator of BYU ScholarsArchive. For more information, please contact ellen_amatangelo@byu.edu. 


\section{Authors}

J. Brent Feland, Sterling C. Hilton, J. Ty Hopkins, lain Hunter, Ulrike H. Mitchell, and J. William Myrer 


\title{
Neurophysiological Reflex Mechanisms' Lack of Contribution to the Success of PNF Stretches
}

\section{Ulrike H. Mitchell, J. William Myrer, J. Ty Hopkins, lain Hunter, J. Brent Feland, and Sterling C. Hilton}

\begin{abstract}
Background and Purpose: Proprioceptive neuromuscular facilitation (PNF) stretches are widely used in athletics and rehabilitation. Although it has been shown that they produce better range-of-motion (ROM) increases than the slow or static stretch, the mechanisms responsible remain an enigma. This study was conducted to determine whether the previously proposed neurophysiological mechanisms of reciprocal inhibition and autogenic inhibition are responsible for the success of PNF stretches. In addition, the authors assessed the existence of the phenomenon of successive induction because it is used to strengthen reciprocal inhibition. Methods: Eighteen subjects 17-44 y performed the PNF stretches contract-relax (CR) and contract-relax, agonist contract (CRAC). EMG data were collected from the medial hamstring muscles via surface and indwelling wire electrodes and analyzed for reciprocal inhibition and successive induction, as well as autogenic inhibition (surface electrodes only). Results: Reciprocal inhibition was not evident. The results indicated an elevated rather than an inhibited EMG during the antagonist contraction, possibly representing cocontraction. The authors did confirm the presence of successive induction. Autogenic inhibition was also not evident, and the expected inhibition and therefore lower EMG values after muscle contraction were not observed; instead, they were higher than baseline. Conclusion: Previous neurophysiological explanations for mechanisms of PNF stretching appear to be inadequate. This study corroborates previous findings that a muscle's tone increases during its antagonist's contraction. Other explanations should be considered regarding the mechanism for the effectiveness of the CRAC and CR PNF techniques in a nonneurologically impaired population.
\end{abstract}

Keywords: proprioceptive neuromuscular facilitation, successive induction, flexibility, stretching

Skilled therapists use techniques that manipulate external input to the central nervous system to obtain optimal treatment outcomes. Proprioceptive neuromuscular facilitation (PNF) comprises patterns and techniques specifically designed to treat neurologically impaired patients. ${ }^{1}$ These techniques and variations thereof were later used in the treatment of orthopedic impairments, and today PNF stretches are widely used in sports medicine. ${ }^{2}$ Two commonly used PNF stretch

Mitchell, Myrer, Hopkins, Hunter, and Feland are with the Dept of Exercise Sciences, and Hilton, the Dept of Educational Leadership and Foundations, Brigham Young University, Provo, UT. 
techniques are contract-relax (CR) and contract-relax, agonist contract (CRAC). During the CR technique the therapist first passively brings the targeted muscle group (the group being stretched) to the point of resistance where further elongation or range of motion (ROM) is restricted. In this position the targeted muscles are isometrically contracted, immediately after which they are stretched to a new point of limitation. The CRAC technique is identical up to this point but adds a contraction of the agonistic muscle group while the therapist stretches the targeted muscle group.

The genesis of PNF was partially based on Sherrington's ${ }^{3}$ findings of reciprocal inhibition and successive induction. These are neurophysiological phenomena, in which the contraction of agonist muscles inhibits or facilitates contraction of their antagonists. Specifically, reciprocal inhibition describes the phenomenon in which while one muscle group is activated, its antagonist is inhibited. It is based on the principle that an isometric contraction increases the firing rate of its own muscle spindles. They in turn send stimuli to Ia-inhibitory interneurons, presumably inhibiting alpha motoneurons of the antagonistic muscles. ${ }^{4,5}$ This leads to relaxation of the antagonistic muscles and/or a depression of the amplitude of the muscle stretch-reflex response. ${ }^{4}$ The CRAC technique is said to take advantage of this occurrence.

Successive induction (the facilitation of the agonist muscle after the antagonist's contraction) also purportedly aids in the success of the CRAC technique: Kabat's ${ }^{6}$ clinical experience confirmed that immediately after voluntary active contraction of the agonist, motion of the antagonist was facilitated. Kabat attributed this to successive induction ${ }^{6}$ and used it to create a PNF strengthening technique that became known as "reversal of antagonists." ${ }^{6,7}$ The primary mechanism behind this technique is postulated to be the autogenic inhibition and reciprocal facilitation action of the Golgi tendon organs (GTOs). ${ }^{8}$ The CRAC technique presumably takes advantage of this by maximizing the force of the second opposing muscle contraction and therefore increasing the coinciding reciprocal inhibition in the target muscle. ${ }^{1}$

Autogenic inhibition is the neurophysiologic phenomenon in which the maximal contraction of a muscle activates the force-sensitive GTOs, which inhibit the alpha motoneurons of the same muscle via Ib-inhibitory interneurons. The CR technique supposedly uses the phenomenon of autogenic inhibition to promote the passive elongation of the target muscle.

Several studies ${ }^{9-14}$ have reported that PNF stretching techniques brought about greater ROM improvements than static or ballistic stretching. It was assumed that PNF stretching techniques relaxed the muscle undergoing stretch by virtue of inhibition, thereby allowing for greater ROM. ${ }^{12,14}$ Using surface electromyography (EMG), Moore and Hutton ${ }^{15}$ investigated the relative level of muscle relaxation achieved during different stretches. They found that the CRAC technique produced not only the greatest ROM increase but also significantly more EMG hamstring activity during the stretch than either the static or CR technique. Subsequent surface EMG studies ${ }^{16-19}$ assessed different stretches and confirmed the apparent paradox of greatest ROM gains coinciding with greatest EMG readings of the muscle being stretched when using PNF stretches. Etnyre and Abraham ${ }^{20}$ applied the CRAC technique using the soleus as the target muscle and tibialis anterior as the antagonistic muscle. They used indwelling wire electrodes, as well 
as surface electrodes. Their surface electrode readings indicated the noted paradox of increased EMG activity of the target muscle during the stretch. The indwelling electrodes, however, indicated reciprocal inhibition of the target muscle during the stretch throughout contraction of the opposing anterior tibialis. The authors suggested that the surface electrodes masked the inhibition by picking up cross talk from the active antagonistic muscles, whereas the wire electrodes did not have this disadvantage and were therefore able to show the actual inhibition. We found no further studies using indwelling wire electrodes to corroborate or refute their findings.

Therefore, the purpose of this study was to investigate the validity of the phenomena of reciprocal inhibition, successive induction, and autogenic inhibition as viable mechanisms to explain the success of PNF stretching.

\section{Design}

\section{Subjects}

We recruited 18 subjects, 16 men and 2 women (age $26.3 \pm 5.9$ years, height 177 $\pm 10.3 \mathrm{~cm}$, body mass $79 \pm 19 \mathrm{~kg}$ ), with tight hamstrings, defined as $20^{\circ}$ or greater loss of full right knee extension $\left(0^{\circ}\right)$ with the right hip at $90^{\circ}$ of flexion and the left leg extended on the treatment table while in the supine position. ${ }^{21}$ The subjects' mean knee flexion in that position was $36^{\circ}\left( \pm 8.7^{\circ}\right)$. A power analysis based on pilot data was used to determine the appropriate number of subjects.

Exclusion criteria were prior history of lower extremity infirmity or pathology within the year before testing, neurological impairments in the lower extremities, osteomyelitis, acute inflammatory joint disease, rheumatoid arthritis and osteoarthritis, advanced osteoporosis, pregnancy, and current intake of medication specifically designed to affect musculoskeletal tissue, such as anti-inflammatory, pain-relief, or arthritis medication. We also excluded subjects who had started a new activity that addressed flexibility, such as dancing or karate, within the month before the study and those not exhibiting tight hamstrings. These criteria were assessed by questioning the subject during the initial screening. Subjects were healthy, uninjured, and normally active. They were asked to maintain their normal activity level for their 1-week participation in the study. All subjects signed a consent form approved by the university institutional review board during an information meeting where they were instructed in the stretching techniques. They were familiarized with the starting position of the stretches and were able to practice maximum voluntary isometric contractions of the hamstrings in that position until they felt comfortable with the procedure. The subjects also practiced producing a hamstring contraction of $89 \mathrm{~N}(20 \mathrm{lb})$ into the investigator's shoulder. These data were collected before each set of trials for later use to normalize the EMG data. During the information meeting, the subjects were scheduled for datacollection sessions the following week.

\section{Instrumentation}

Muscle activity was measured using the BIOPAC MP100 system (BIOPAC Systems Inc, Santa Barbara, CA). Signals were amplified (TEL 100M, BIOPAC 
Systems) from reusable surface-contact electrodes (TSD150). The indwelling electrodes were made by the primary investigator after the method described by Basmajian and De Luca. ${ }^{22}$ We used Stablohm 800 H-poly nylon green wire (California Fine Wire Co, Grover Beach, CA) and 27-gauge 4.9-cm-long hypodermic needles. The EMG measurements were collected at $1000 \mathrm{~Hz}$. The input impedance of the amplifier was $1.0 \mathrm{M} \Omega$, with a common-mode rejection ratio of $90 \mathrm{~dB}$; high- and low-pass filters of 20 and $400 \mathrm{~Hz}$, respectively; a signal-tonoise ratio of $70 \mathrm{~dB}$; and a gain of 1000 . Raw EMG signals were processed using a root-mean-square algorithm with a 10-millisecond moving window with AcqKnowledge Software (version 3.73, BIOPAC Systems). A handheld dynamometer (microFET2, Hoggan Health Industries, Draper, UT) was used to obtain data for EMG normalization. A standard plastic goniometer was used to measure ROM at the knee and hip.

\section{Testing Protocol}

Overview. After warm-up, the subjects performed the 2 PNF stretching techniques, $\mathrm{CR}$ and $\mathrm{CRAC}$, in random order with at least 1 day between techniques. EMG data of the target and antagonistic muscles were collected and compared within trials to determine whether successive induction, reciprocal inhibition, and autogenic inhibition occurred.

Electrode Placement. Shaving was performed when deemed necessary. The skin was lightly debrided and cleansed with an alcohol pad. Adhesive surface electrodes were placed on the skin using conducting gel with an interelectrode distance of $2 \mathrm{~cm}$ in the direction of muscle-fiber orientation. The fibers of the vastus lateralis run obliquely from proximal lateral to distal medial, and the fibers of the medial hamstrings run longitudinally along the femur. The placement of the surface electrodes was found by palpating the greatest muscle bulk of the vastus lateralis and medial hamstrings during contraction. For the vastus lateralis this was approximately $10 \mathrm{~cm}$ superior to the base of the patella; for the medial hamstrings it was approximately halfway between the ischial tuberosity and the popliteal fossa. The surface electrodes were traced with a pen so the same electrode placement could occur the next data-collection day. A 27-gauge hypodermic needle with wire electrodes resting inside was inserted into the appropriate muscle belly. The needle was removed, leaving behind the flexible wire electrodes, which were taped to the skin. After collecting the data, the wire electrodes were removed by gently pulling them out. For the cases in which both surface and wire electrodes were needed, the needle electrode was placed $3 \mathrm{~mm}$ distal to the surface electrode. The insertion sites were marked with a pen. The surface ground electrode was located on the medial malleolus of the left leg. All electrode placements were checked using palpation and EMG activity.

Dynamometer Placement. The placement of the dynamometer was kept consistent over the days of data collection by positioning it 5 in $(\sim 13 \mathrm{~cm})$ proximal to the heel, at $90^{\circ}$ to the lower leg.

Normalization. Before the collection of the normalization data the subjects warmed up for the stretches by riding a stationary bicycle ergometer (Monarch 
818E, Stockholm, Sweden) at $75 \mathrm{~W}$ at a comfortable pace (60 to $70 \mathrm{RPM}$ ) for 5 minutes. For the normalization we used an isometric reference position, which was the same as the starting position for the stretch (Figure 1). The subject pushed for 5 seconds into the therapist's shoulder with $89 \mathrm{~N}$ force, as measured by a handheld dynamometer (microFET2, Hoggan Health Industries, Draper, UT). Two seconds of processed EMG data within those 5 seconds (seconds 2 and 3 ) were used for normalization. All EMG data were reported as ratios of stretch to reference EMG (Tables 1 and 2).

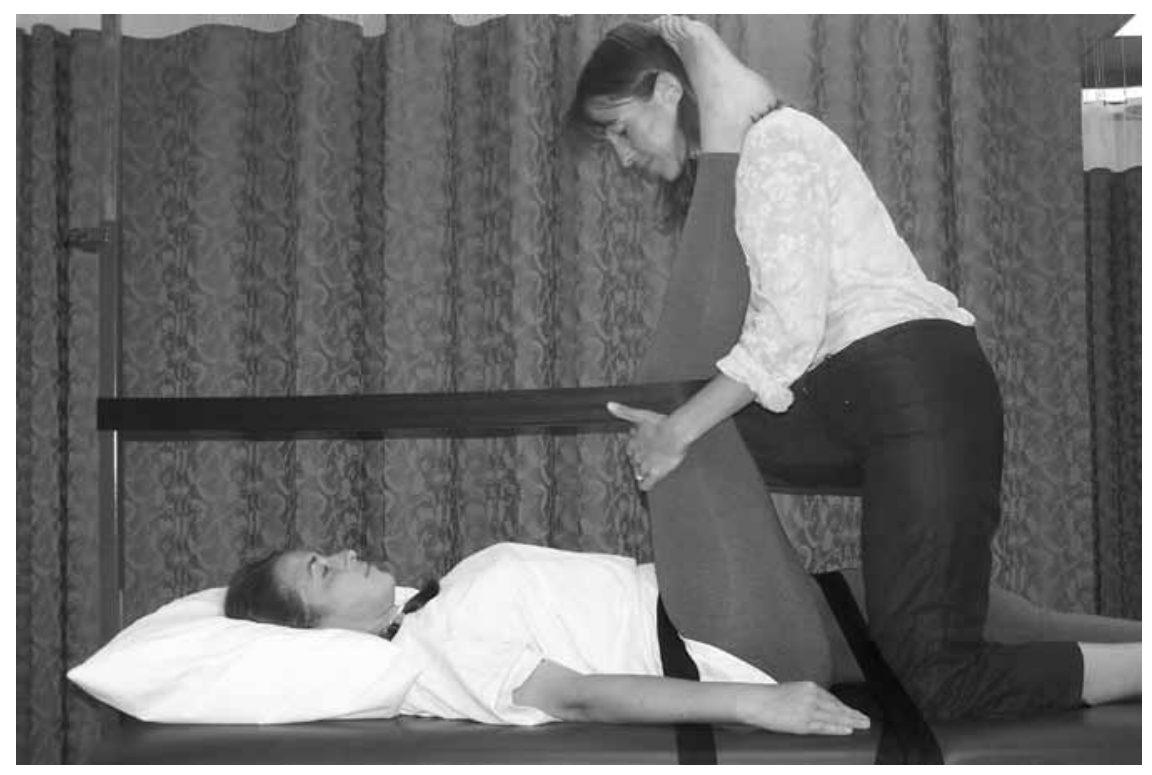

Figure 1 - Starting position for the slow, contract-relax, and contract-relax, agonist contract stretches.

\section{Table 1 Data for Reciprocal Inhibition}

\begin{tabular}{lllcc}
\hline & $\boldsymbol{F}_{\mathbf{1 , 1 6}}$ & $\boldsymbol{P}$ & Time 1, mean (SD) & Time 2, mean (SD) \\
\hline Hamstrings (surface) $^{\mathrm{a}}$ & 0.01 & .93 & $0.63( \pm 0.28)$ & $0.62( \pm 0.27)$ \\
Hamstrings (wire) $^{\mathrm{a}}$ & 2.63 & .13 & $0.15( \pm 0.21)$ & $0.23( \pm 0.3)$ \\
Quadriceps (surface) $^{\mathrm{b}}$ & 0.85 & .37 & $1.84( \pm 2.7)$ & $1.35( \pm 0.85)$ \\
Quadriceps (wire) $^{\mathrm{b}}$ & 0.82 & .38 & $3.84( \pm 12.72)$ & $4.01( \pm 12.67)$ \\
\hline
\end{tabular}

Time 1: agonist (site of EMG measurements) and antagonist at rest. Time 2: agonist at rest, antagonist contracting maximally.

a During contract-relax, agonist contract.

${ }^{\mathrm{b}}$ During contract-relax. 


\section{Table 2 Data for Successive Induction and Postactivation Potentiation}

\begin{tabular}{|c|c|c|c|c|}
\hline & \multicolumn{2}{|c|}{$\begin{array}{c}F \text {-Test and } P \text { Values } \\
\text { for Time in the ANOVA } \\
\text { Model }\end{array}$} & \multicolumn{2}{|c|}{ EMG Data (Normalized) } \\
\hline & $F_{1,16}$ & $P$ & $\begin{array}{c}\text { Quads } \\
\text { contraction 1, } \\
\text { mean (SD) }\end{array}$ & $\begin{array}{c}\text { Quads } \\
\text { contraction 2, } \\
\text { mean (SD) }\end{array}$ \\
\hline $\begin{array}{l}\text { Successive induction (with } \\
\text { hamstring contraction) }\end{array}$ & 11.92 & .003 & $5.61( \pm 4.6)$ & $7.56( \pm 6.47)$ \\
\hline $\begin{array}{l}\text { Postactivation potentiation } \\
\text { (without hamstring } \\
\text { contraction) }\end{array}$ & 1.82 & .2 & $6.93( \pm 7.9)$ & $8.37( \pm 11.53)$ \\
\hline
\end{tabular}

Stretching Technique. For the starting position (Figure 1), subjects were positioned supine on a padded examination table with the right hip flexed to $110^{\circ}$ as measured by a standard goniometer. The goniometer's arms were placed parallel to the long axis of the femur and lumbar spine with its axis of rotation placed over the lateral aspect of the hip joint. This position was maintained by a 5 -cm-wide strap that securely tied the thigh to a post. The left thigh was strapped to the table to stabilize the pelvis. The pelvis was also stabilized directly by a belt. The investigator, a physical therapist, kneeled in front of the subject, the subject's right lower leg resting on the therapist's shoulder. The therapist held the subject's thigh with both hands and extended the subject's right lower leg to a point of restriction, where the hamstrings became tightened. This point was determined through a combination of verbal feedback from the subject indicating tightness, but not pain, and a clinical soft-tissue end feel detected by the investigator. ${ }^{23}$

For the CR technique the therapist brought the subject's right leg to the point of hamstring restriction. The subject then actively maximally contracted the hamstrings for 6 seconds $^{24}$ toward the therapist's shoulder (phase 1). A recent study ${ }^{25}$ showed that there is no difference in flexibility gains between $20 \%, 60 \%$, or $100 \%$ of maximal voluntary isometric contractions. However, because we do not know the relationship between ROM gains and degree of inhibition or facilitation, we used maximal resistance to benefit from maximal reflex activity. ${ }^{6}$ Immediately after the isometric contraction the therapist passively stretched the hamstrings for 10 seconds (phase 2). This stretch was performed 4 times with 20 seconds between trials. Occurrence of reciprocal inhibition in the quadriceps muscles and of autogenic inhibition in the medial hamstrings were assessed during this stretch technique.

For the CRAC technique the subject's right leg was brought to the same point of hamstring restriction. The actual stretch began with an active 5-second quadriceps contraction, immediately followed by a maximal hamstring contraction into the therapist's shoulder, which was followed by a second quadriceps contraction. The latter contraction served as an active assistance to the stretch by the subject. The durations of the hamstring contraction and the stretch were 6 and 10 seconds, respectively. Quadriceps EMG measurements were taken for the duration of the trial. These data were used to determine whether there was successive induction 
in the quadriceps. Existence of reciprocal inhibition in the hamstrings was also assessed. This stretch was performed 4 times with 20 seconds rest between trials. All manual stretch assistance was provided by the same therapist.

The subjects also performed 2 consecutive contractions of the quadriceps without a hamstring contraction between CRAC stretches. Surface EMG measured quadriceps contraction activity to assess whether any second quadriceps contraction was stronger than the first one.

Knee ROM was measured to make sure that a stretch in fact took place and that the knee extended as a result of the stretch. The goniometer's arms were placed parallel to the long axis of the femur and tibia with its axis of rotation over the lateral aspect of the knee joint.

\section{Dependent Variables}

This study was limited to examining the effects of 2 specific PNF stretches on the hamstrings and quadriceps muscles. Because the exact duration of inhibition or facilitation caused by muscle contraction is not known we decided to examine a 2 -second window to evaluate the presence of reciprocal inhibition, autogenic inhibition, and successive induction. We chose this time frame because we felt that an interval less than this would be of no clinical consequence in regard to enhancing a stretch or increasing muscle contraction.

$\Delta \mathrm{EMG}_{1}$ measured reciprocal inhibition in the hamstrings during the CRAC technique (Figure 2). Hamstring EMG for 2 seconds before quadriceps contraction (time 1) and for 2 seconds after the beginning of quadriceps contraction (time 2) were compared. Reciprocal inhibition was considered present when hamstring activity, as measured by wire and/or surface electrodes, was statistically lower during maximal quadriceps activation than at baseline ( 2 seconds before contraction).

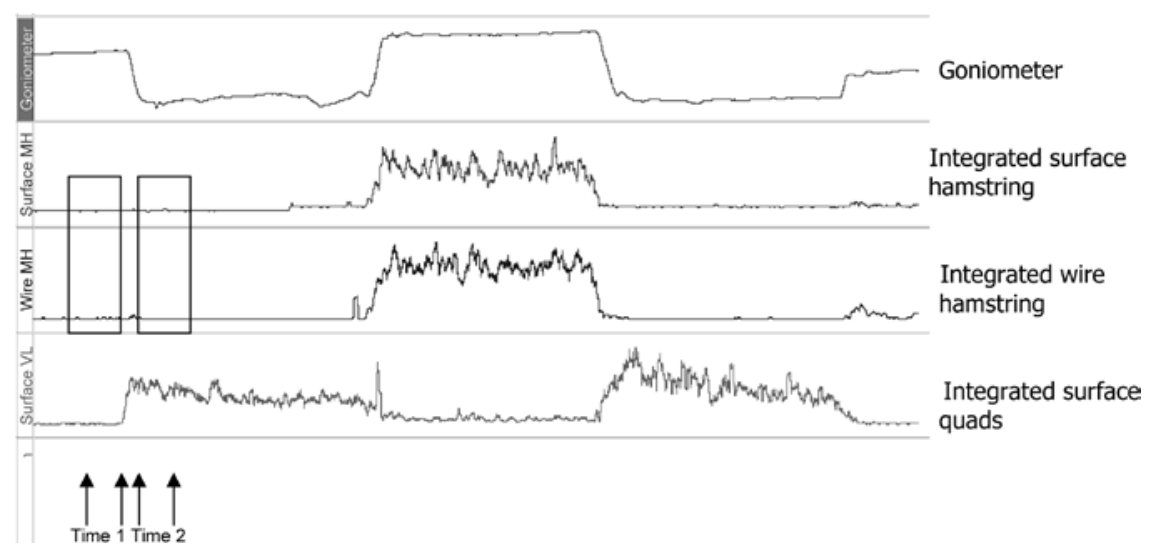

Figure 2 - Reciprocal inhibition (hamstrings shown). 
$\Delta \mathrm{EMG}_{2}$ measured reciprocal inhibition in the quadriceps during the $\mathrm{CR}$ technique via surface and indwelling wire electrodes. Quadriceps EMG of 2 seconds before hamstring contraction (time 1) and 2 seconds after the beginning of hamstring contraction (time 2) were compared. Reciprocal inhibition was considered present when quadriceps activity was statistically lower during maximal hamstring activation than when the hamstrings were not actively contracted.

$\Delta \mathrm{EMG}_{3}$ measured successive induction (Figure 3), or facilitated contraction of the agonist, after contraction of the antagonistic muscle during the CRAC technique. It was measured via surface electrodes. It was the difference between the 2 -second mean quadriceps contraction EMG before the hamstring contraction and the 2-second mean quadriceps contraction EMG after it. Successive induction was considered present if the ratio of the quadriceps EMG after hamstring contraction was statistically higher than the ratio of the quadriceps contraction before hamstring contraction.

$\Delta \mathrm{EMG}_{4}$ via surface electrodes was used to determine whether any second contraction was stronger than the first one, without a contraction of the antagonist in between.

$\Delta \mathrm{EMG}_{5}$ measured autogenic inhibition (Figure 4), or facilitated relaxation, after maximal contraction of the same muscle during the CR technique. It was measured via surface electrodes in the right medial hamstring muscle. The mean hamstring EMG of 2 seconds immediately after the isometric contraction and the mean hamstring EMG 2 seconds before (baseline) contraction were compared. Inhibition was considered present if the mean hamstring EMG immediately after the isometric contraction was statistically significantly lower than the hamstring EMG before (baseline).

An alpha level of .05 was set for $\Delta \mathrm{EMG}_{1-5}$.

Custom software, written in Visual Basic, was used to find the beginning and end of each contraction. The routine then calculated the average root mean square

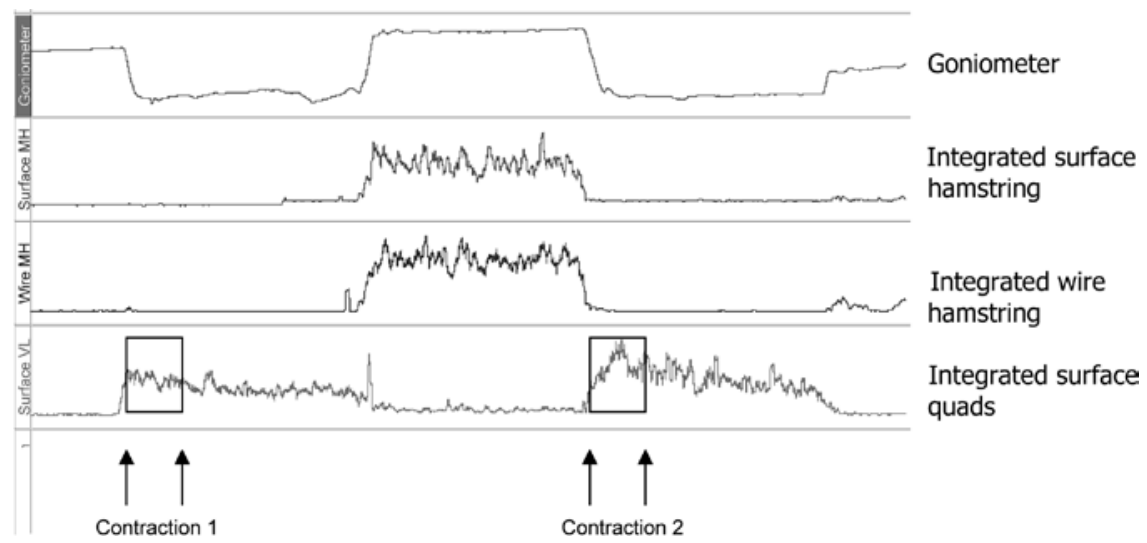

Figure 3 - Successive induction (shown with target muscle contraction). 


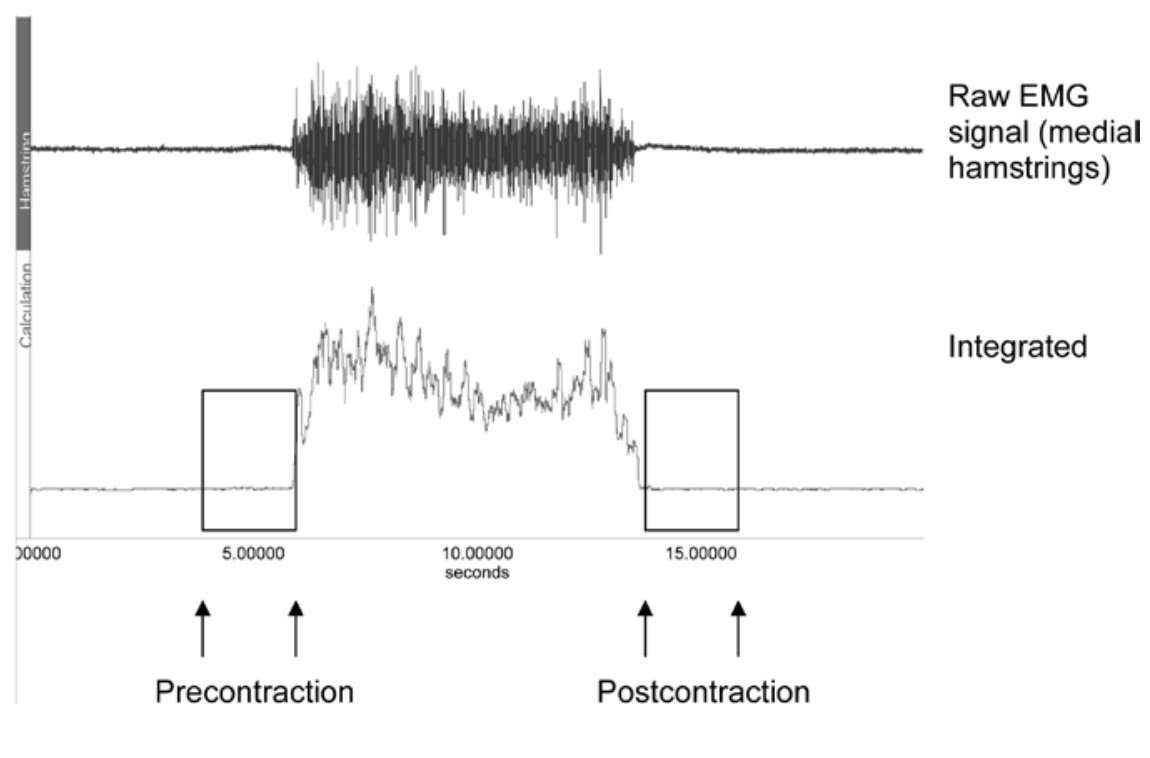

Figure 4 - Autogenic inhibition.

of each specific 2-second segment, depending on the examined variable. Random manual checks ensured proper sampling of the EMG time segments. These data were normalized, yielding a ratio. After this, the program computed the differences in precontraction, postcontraction, and during-contraction ratios, depending on the examined variable. The investigator reviewed each data point to subjectively verify that the data were free from artifact and marked appropriately.

\section{Data Analysis}

This was a crossover experimental research study, using a $2 \times 4$ (time $\times$ order) factorial design for each outcome. The independent variables were time ( 2 levels: pre and during) and order (4 levels: trials 1 to 4 ); the dependent variables were differences in EMG readings that could indicate reciprocal inhibition in the hamstrings $\left(\Delta \mathrm{EMG}_{1}\right)$, differences in EMG readings that could indicate reciprocal inhibition in the quads $\left(\Delta \mathrm{EMG}_{2}\right)$, differences in EMG readings that could indicate successive induction $\left(\Delta \mathrm{EMG}_{3}\right)$, differences in EMG readings that show that any second contraction was stronger than the first one $\left(\Delta \mathrm{EMG}_{4}\right)$, and differences in EMG readings that could indicate autogenic inhibition $\left(\Delta \mathrm{EMG}_{5}\right)$.

\section{Dependent-Variable $\Delta$ EMG}

We checked for a significant interaction between the fixed effects time and order and for a significant order effect for reciprocal inhibition, successive induction, and autogenic inhibition. We found none. We therefore tested the null hypothesis $\mu_{\text {Pre }}=\mu_{\text {Post }}$ versus the alternative hypothesis that they are different. 


\section{Results}

Before final data analysis was performed outliers were excluded from the data pool. An outlier was defined as a data point lying at least 3.5 group SDs beyond the subject's mean. The normalized data represent the factor by which the EMG data of the reference position were multiplied.

Reciprocal inhibition (Table 1) was measured 4 different ways. During the CRAC technique we checked possible inhibition in the hamstrings using wire (8 outliers in 60 available data points) and surface electrodes (1 outlier in 67 data points); during the CR technique we used wire ( 3 outliers in 67 data points) and surface electrodes (no outlier in 66 data points) to check for reciprocal inhibition in the quadriceps.

Reciprocal inhibition was not observed in either muscle group or electrode type. Considerably higher variability was noted with the wire electrodes than surface electrodes in the quadriceps.

Successive induction (Table 2) was measured during the 2 quadriceps contractions, with a hamstring contraction between, during the CRAC technique (no outliers). To show that not just any second contraction is significantly stronger than the first contraction we also compared 2 quadriceps contractions without a separating hamstring contraction, merely a relaxation phase between (contract, relax, contract). This is considered a form of postactivation potentiation ${ }^{26}$ (Table 2 ). In both conditions we found greater values for the second quadriceps contraction, but only in the CRAC condition was it statistically significant $(P=$ $.003)$.

We did not observe autogenic inhibition. Autogenic inhibition was considered present if the average of normalized root-mean-square integrated EMG data 2 seconds after the muscle's contraction was significantly smaller than the average of 2 seconds immediately before the contraction (no outliers). During the time after hamstring contraction the hamstring was subjected to a slow stretch (phase 2 of the CR stretch). Our data exhibited a significantly higher $\left(F_{1,17}=7.19, P=\right.$ .016) EMG amplitude postcontraction than precontraction for the CR technique.

The mean ROM increase for the 4 trials in the CR technique was $17.36^{\circ}( \pm$ $10.79^{\circ}$ ), and the CRAC technique produced a mean ROM increase of $10.07^{\circ}( \pm$ $\left.4.25^{\circ}\right)$.

\section{Discussion}

The success of PNF stretches has largely been attributed to neurophysiologic mechanisms. ${ }^{9,13,14,27,28}$ Most of them are credited to the muscle spindle and the GTOs and their reflex activity: Activation of the muscle spindle elicits contraction of the agonist and inhibition of the antagonist, sometimes referred to as the myotatic reflex or stretch reflex. ${ }^{5}$ Activation of GTOs elicits inhibition of the agonist (autogenic inhibition, or inverse myotatic reflex ${ }^{5,29}$ ) while facilitating the antagonist. ${ }^{12,30}$ The first study to cast doubt on the neurophysiological theory behind PNF techniques attempted to measure stretch-induced inhibition via EMG. ${ }^{15}$ To the authors' surprise, they found the opposite: facilitated muscles as evidenced in increased EMG activity during the time the muscle was being stretched. Other studies agreed. ${ }^{16,18}$ 
Several years later Etnyre and Abraham ${ }^{20}$ refuted those findings. Our study's purpose therefore was to clarify several aspects of PNF techniques: Can the phenomenon of reciprocal inhibition in connection with PNF stretches be confirmed using surface and/or wire electrodes? Can successive induction be recorded? Can autogenic inhibition be shown?

\section{Reciprocal Inhibition}

We did not observe reciprocal inhibition using surface or wire electrodes. If reciprocal inhibition had occurred, the EMG amplitude of the noncontracting muscle should have been lower during its antagonist's contraction compared with baseline. This did not occur. We found that a coinciding contraction of the opposing muscles increased target-muscle EMG surface and fine-wire values when compared with baseline, confirming the findings of several other authors. ${ }^{15-19}$

We believe that the increase in EMG values was caused by the coactivation of antagonistic muscles during the stretching procedure. Coactivation occurs throughout the body and is present during all activities that require stabilization. In our study we examined 2 muscles that span 2 joints, the knee and the hip. Although the knee was flexing slightly during contraction of the target muscle and extended during the stretching procedure, the hip needed to be stabilized. This required the hamstrings and quadriceps to work as true synergists and cocontract. Coactivation of the hamstrings and quadriceps during the CRAC technique enhanced the ability of the leg to change direction. The CRAC technique uses alternating movements, which allows the coactivation of the involved muscles to accommodate better than if the antagonist muscles were fully relaxed before contraction. ${ }^{31}$

Etnyre and Abraham ${ }^{20}$ evaluated the paradox of increased EMG amplitudes in the stretching muscle during PNF stretches while yielding the greatest ROM gains compared with static stretching. They found that reciprocal inhibition could be shown by using indwelling wire EMG electrodes. They consequently suggested that earlier studies using surface electrodes that showed an increase in muscle activity instead of inhibition might have picked up cross talk, masking the inhibition. We did not confirm that assertion. Our wire electrode readings in the noncontracting muscle exhibited a trend toward higher EMG values when the antagonist was contracting than when both were at rest. At the same time the surface electrodes demonstrated a slight, yet not significant, decrease in activity during antagonist contraction (Table 1). These findings follow Winter's ${ }^{32}$ clarification on possible cross-talk contamination. Winter submits that any electrode has a pickup range of about $2 \mathrm{~cm}$. For the surface electrode this translates to a spherical volume under the skin surface, and for the indwelling electrode this means a sphere of $2-\mathrm{cm}$ radius. Winter concludes that a surface electrode is less prone to pick up cross talk than an indwelling electrode several centimeters below the surface. Our results agree with those of Winter. The wire EMG exhibited a higher, though not significantly, amplitude during antagonist contraction (time 2) than at rest (time 1) compared with the surface electrodes, which demonstrated slightly lower values during antagonist contraction than at rest. 


\section{Successive Induction}

To assess successive induction, the subjects performed 4 trials. For each trial they voluntarily contracted the quadriceps maximally twice with a hamstring contraction between. To give more strength to our findings we employed another, contrasting paradigm. The subjects were asked to maximally contract the quadriceps, relax, and then contract the quadriceps again. This model revealed whether any second contraction of the quadriceps muscle was enhanced compared with the first.

Our results did show successive induction, or facilitated quadriceps contraction, after contraction of the antagonist medial hamstrings. During the CRAC technique the subject's second quadriceps contraction, which followed a hamstring contraction, exhibited greater EMG amplitude than the first one, which did not have a preceding hamstring contraction. Our study, therefore, supports the use of the PNF technique "reversal of antagonists," 6,7 because it facilitates muscle contraction. It does not, however, support the notion that with that greater quadriceps contraction there is greater reciprocal inhibition in the hamstrings. Comparison of the 2 sets of hamstring EMG data (during the first and during the second quadriceps contraction) shows no statistical difference between the 2 groups and therefore no inhibition.

We found 3 studies ${ }^{33-35}$ that investigated successive induction. Two of them ${ }^{34,35}$ evaluated a potential strengthening component and decrease in fatigue that, according to the authors, should have occurred after training under "successive induction conditions." In both studies the authors found no strength increase as a result of the training. The big difference between those 2 studies and ours is that we expected the greater EMG amplitude to occur immediately after the antagonistic contraction, not after several trials and training sessions. The leap that those authors made when they concluded that greater EMG amplitude immediately after an antagonistic contraction should mean an actual measurable strength increase seems to be flawed. The third and oldest study ${ }^{33}$ did demonstrate successive induction. That study evaluated the presence of increased EMG amplitude immediately after the contraction of the antagonist, similar to our study. It seems plausible that successive induction is an event of short duration. The facilitation achieved by successive induction is widely incorporated in the treatment of neurologically impaired or weakened patients in physical therapy. ${ }^{8}$ Perhaps this facilitation is not large enough to achieve strength gains in a healthy population.

We also found that any second quadriceps contraction was higher than the first one, but only a second quadriceps contraction with a preceding hamstring contraction was statistically significantly higher $(P=.003)$. Our data seem to support Kabat's ${ }^{6}$ use of Sherrington's ${ }^{3}$ findings of successive induction (Table 2 ).

More recently the phenomenon of the second contraction's being stronger than the first one has been termed postactivation potentiation. ${ }^{26}$ Although we found an increased EMG amplitude on the second quadriceps contraction, the mechanism proposed to date for postactivation potentiation is biochemical rather neurological. ${ }^{26}$

\section{Autogenic Inhibition}

We were unable to show autogenic inhibition. Tanigawa ${ }^{36}$ found large ROM increases in a PNF stretch group compared with a control group and suggested 
that it might be the result of autogenic inhibition. No physiologically based data were collected during that study to corroborate that notion. Houk and Henneman ${ }^{37}$ investigated the sensitivity of GTOs in the cat by electrically stimulating different filaments of the ventral root. No stretch was performed. The authors confirmed the existence of autogenic inhibition and referred to it as the "inverse myotatic reflex." In our study, we found that the EMG amplitude of the contracting muscle was higher during the 2 seconds after the contraction than at baseline before the contraction. Two arguments can be found in the literature that contradict the idea that activated GTOs bring about lasting muscle inhibition. First, GTOs cease or at least decrease firing as soon as the muscle relaxes after contraction. ${ }^{38,39}$ One study ${ }^{40}$ reports that the inhibitory effect declined even during contraction. Thus, although the GTO might be able to inhibit its muscle, the short duration of the inhibition makes it unlikely that it enhances the therapeutic stretch of a muscle. Second, the motor response of an activated GTO is not limited to the same muscle but can also affect its synergists and even antagonists. ${ }^{41}$ Moreover, the motor response could also be excitatory, not just inhibitory. It is therefore not reasonable to attribute a certain motor response to activated GTOs. The increased EMG amplitude of the contracting muscle after the contraction compared with before the contraction could be a result of enhanced musclespindle postcontraction discharge activity. ${ }^{38,42}$ Wilson et al ${ }^{38}$ examined alterations in discharge rate of muscle-spindle afferents after isometric contractions. They found that in their population of 55 spindle afferents the mean postcontraction discharge rate was $65 \%$ higher than the mean precontraction discharge rate. One possible explanation for this phenomenon is found in Hutton et al's ${ }^{42}$ article: "The portion of the intrafusal fiber underlying the sensory wrapping fails to return to its pre contraction length, leaving the ending under persisting stretch" (p. 1101). The cause of the muscle spindle's inability to return to a normal state is hypothesized to lie in persisting cross-bridge formations between actin and myosin myofilaments in the intrafusal fiber. ${ }^{42}$ This intrafusal fiber stretch leads to contraction of the muscle fiber ${ }^{42}$ expressed in greater EMG amplitude. Although this is a credible explanation for increased postcontraction muscle activity, it does not transfer to a muscle undergoing stretch immediately after contraction. A stretch breaks the cross-bridge formations and returns the muscle spindle's discharge rate to its precontraction state..$^{38,42}$

\section{Conclusion}

The traditional mechanisms of PNF proposed by Knott and Voss ${ }^{1}$ and $\mathrm{Kabat}^{6}$ cannot be transferred from neurologically impaired patients to a normal population. Neurophysiological factors such as reciprocal inhibition and autogenic inhibition appear to not be responsible for the higher ROM gains achieved through PNF stretching. Our study contested the notion that reciprocal inhibition could be shown using indwelling wire electrodes when performing the CRAC technique. ${ }^{20}$ The findings of multiple researchers ${ }^{15-19}$ that a muscle's tone increases during its antagonist's contraction seems to be validated by this study. Successive induction, the technique used to facilitate muscle contraction of the opposing muscle and presumably to promote reciprocal inhibition of the target muscle during the CRAC 
technique, was confirmed by our study. However, although successive induction did occur, it was not strong enough to bring about an increase in reciprocal inhibition.

\section{References}

1. Knott M, Voss D. Proprioceptive Neuromuscular Facilitation: Patterns and Techniques. New York, NY: Paul Hoeber; 1956.

2. Surburg P, Schrader J. Proprioceptive neuromuscular facilitation techniques in sports medicine: a reassessment. J Athl Train. 1997;32(1):34-39.

3. Sherrington C. The Integrative Action of the Nervous System. New Haven, CT: Yale University Press; 1906.

4. Chalmers G. Re-examination of the possible role of Golgi tendon organ and muscle spindle reflexes in proprioceptive neuromuscular facilitation muscle stretching. Sports Biomech. 2004;3(1):159-183.

5. Plowman S, Smith D. Neuromuscular aspects of movement. In: Lupash E, Klingler AM, eds. Exercise physiology for health, fitness, and performance. 2nd ed. San Francisco, CA: Benjamin Cummings; 2002:566-589.

6. Kabat H. Proprioceptive facilitation in therapeutic exercise. In: Licht S, ed. Therapeutic Exercise. 2nd ed. New Haven, CT: Elizabeth Licht; 1961:327-343.

7. Kabat H. Studies on neuromuscular dysfunction: XV. the role of central facilitation in restoration of motor function in paralysis. Arch Phys Med. 1952;33:521-533.

8. Sullivan P, Markos P, Minor M. An Integrated Approach to Therapeutic Exercise: Theory \& Clinical Application. Reston, VA: Reston; 1982:128-160.

9. Sady SP, Wortman M, Blanke D. Flexibility training: ballistic, static or proprioceptive neuromuscular facilitation? Arch Phys Med Rehabil. 1982;63:261-263.

10. Etnyre B, Lee E. Chronic and acute flexibility of men and women using three different stretching techniques. Res Q Exerc Sport. 1988;59(3):222-228.

11. Etnyre B, Abraham L. Gains in range of ankle dorsiflexion using three popular stretching techniques. Am J Phys Med. 1986;65(4):189-196.

12. Prentice WE. A comparison of static stretching and PNF stretching for improving hip joint flexibility. J Athl Train. 1983;18(1):56-59.

13. Holt LE, Travis TM, Okita T. Comparative study of three stretching techniques. Percept Mot Skills. 1970;31:611-616.

14. Guissard N, Duchateau J, Hainaut K. Muscle stretching and motoneuron excitability. Eur J Appl Physiol. 1988;58:47-52.

15. Moore MA, Hutton RS. Electromyographic investigation of muscle stretching techniques. Med Sci Sports Exerc. 1980;12(5):322-329.

16. Osternig LR, Robertson R, Troxel R, Hansen P. Muscle activation during proprioceptive neuromuscular facilitation (PNF) stretching techniques. Am J Phys Med. 1987;66(5):298307.

17. Osternig LR, Robertson R, Troxel R, Hansen P. Differential responses to proprioceptive neuromuscular facilitation (PNF) stretch techniques. Med Sci Sports Exerc. 1990;22(1):106-111.

18. Condon SM, Hutton RS. Soleus muscle electromyographic activity and ankle dorsiflexion range of motion during four stretching procedures. Phys Ther. 1987;67(1):2430.

19. Ferber R, Osternig L, Gravelle D. Effect of PNF stretch techniques on knee flexor muscle EMG activity in older adults. J Electromyogr Kinesiol. 2002;12:391-397.

20. Etnyre BR, Abraham LD. Antagonist muscle activity during stretching: a paradox reassessed. Med Sci Sports Exerc. 1988;20:285-289. 
21. Feland J, Myrer J, Schulthies S, Fellingham G, Measom G. The effect of duration of stretching of the hamstring muscle group for increasing range of motion in people aged 65 years or older. Phys Ther. 2001;81-5:1110-1117.

22. Basmajian J, De Luca C. Muscles Alive. 5th ed. Baltimore, MD: Williams \& Wilkins; 1985:19-64.

23. Hayes K, Petersen C. Reliability of assessing end-feel and pain and resistance sequence in subjects with painful shoulders and knees. J Orthop Sports Phys Ther. 2001;31(8):432-445.

24. Nelson K, Cornelius W. The relationship between isometric contraction durations and improvement in shoulder joint range of motion. J Sports Med Phys Fitness. 1991;31$3: 385-388$.

25. Feland J, Marin H. Effect of submaximal contraction intensity in contract-relax proprioceptive neuromuscular facilitation stretching. Br J Sports Med. 2004;38(4):E18.

26. Hamada T, Sale D, MacDougall J, Tarnopolsky M. Postactivation potentiation, fiber type, and twitch contraction time in human knee extensor muscles. $J$ Appl Physiol. 2000;88:2131-2137.

27. Etnyre BR, Kelley BD. Electromyographic analysis of movement following a maximal isometric contraction. Electromyogr Clin Neurophysiol. 1989;29:221-225.

28. Markos PD. Ipsilateral and contralateral effects of proprioceptive neuromuscular facilitation technique on hip motion and electromyographic activity. Phys Ther. 1979;59(11):1366-1373.

29. Granit R. Autogenic inhibition. Electroencephalogr Clin Neurophysiol. 1950;2$4: 417-424$.

30. Enoka RM. Single-joint system components. In: Enoka RM. Neuromechanics of Human Movement. 3rd ed. Champaign, IL: Human Kinetics; 2002:211-240.

31. Enoka R. Multi-joint systems. In: Enoka RM. Neuromechanics of Human Movement. 3rd ed. Champaign, IL: Human Kinetics; 2002:313-358.

32. Winter D. Electromyogram recording, processing, and normalization: procedures and considerations. J Hum Muscle Perform. 1991;1-2:5-15.

33. Holt LE, Kaplan HM, Okita TY, Hoshiko M. The influence of antagonistic contraction and head position on the responses of agonistic muscles. Arch Phys Med Rehabil. 1969;50:279-283.

34. Gabriel D, Kroll W. Successive induction effects upon maximal isometric strength. Clin Kinesiol. 1991;45(3):3-10.

35. Kroll W. Isometric strength and endurance under successive induction conditions. Am Corr Ther J. 1972;26(5):127-131.

36. Tanigawa M. Comparison of the hold-relax procedure and passive mobilization on increasing muscle strength. Phys Ther. 1972;52(7):725-735.

37. Houk J, Henneman E. Responses of Golgi tendon organs to active contractions of the soleus muscle of the cat. J Neurophysiol. 1967;30:466-489.

38. Wilson LR, Gandevia SC, Burke D. Increased resting discharge of human spindle afferents following voluntary contractions. J Physiol. 1995;488(3):833-840.

39. Edin B, Vallbo A. Dynamic response of human muscle spindle afferents to stretch. $J$ Neurophysiol. 1990;63-6:1297-1306.

40. Zytnicki D, Lafleur J, Horcholle-Bossavit G, Jami L. Reduction of Ib autogenic inhibition in motoneurons during contractions of an ankle extensor muscle in the cat. $J$ Neurophysiol. 1990;64-5:1380-1389.

41. Pratt C. Evidence of positive force feedback among hindlimb extensors in the intact standing cat. J Neurophysiol. 1995;73-6:2578-2583.

42. Hutton RS, Smith JL, Eldred E. Postcontraction sensory discharge from muscle and its source. J Neurophysiol. 1973;36:1090-1103. 